\title{
CONTROLE QUÍMICO DE PARREIRA-BRAVA (Cissampelos glaberrima)
}

\author{
Edson D. de Mattos, Pedro Luís da Costa A. Alves, Fabíola V. Môro, José Custódio de Souza, \\ José V. F. Martins e Francisco Rigler Neto
}

\section{ERRATA}

No trabalho citado acima, publicado na REVISTA BRASILEIRA DE HERBICIDAS, v.2, n. 1/2, p. 57-64, 2001, a Tabela 7 (p. 63) apresentou os mesmos dados contidos na Tabela 6. Os dados corretos da Tabela 7 são os seguintes:

Tabela 7. Número de brotações viáveis por rizoma e de rizomas viáveis de parreira-brava, nas profundidades de 30 e $55 \mathrm{~cm}$, em resposta aos tratamentos. Jaboticabal, 2000.

\begin{tabular}{|c|c|c|c|c|}
\hline \multirow{2}{*}{ Tratamento } & \multicolumn{2}{|c|}{ B rotações viáveis } & \multicolumn{2}{|c|}{ Rizomas viáveis } \\
\hline & $30 \mathrm{~cm}$ & $55 \mathrm{~cm}$ & $30 \mathrm{~cm}$ & $55 \mathrm{~cm}$ \\
\hline 2,4-D/picloram & $0,33 b^{1}$ & $2,68 \mathrm{a}$ & $1,13 \mathrm{a}$ & $1,41 \mathrm{a}$ \\
\hline $2,4-\mathrm{D} /$ picloram & $0,00 \mathrm{~b}$ & $2,85 \mathrm{a}$ & $1,41 \mathrm{a}$ & $1,41 \mathrm{a}$ \\
\hline $2,4-\mathrm{D} / \mathrm{picloram}$ & $0,00 \mathrm{~b}$ & $2,57 \mathrm{a}$ & $1,13 \mathrm{a}$ & $1,41 \mathrm{a}$ \\
\hline 2,4-D/picloram+imazapyr & $0,33 \mathrm{~b}$ & $2,40 \mathrm{a}$ & $1,27 \mathrm{a}$ & $1,41 \mathrm{a}$ \\
\hline $2,4-\mathrm{D} / \mathrm{p}$ icloram +imazapyr & $1,00 \mathrm{ab}$ & $2,21 \mathrm{a}$ & $1,27 \mathrm{a}$ & $1,41 \mathrm{a}$ \\
\hline 2,4-D/picloram+imazapyr & $0,00 \mathrm{~b}$ & $2,58 \mathrm{a}$ & $1,27 \mathrm{a}$ & $1,41 \mathrm{a}$ \\
\hline $2,4-\mathrm{D} / \mathrm{picloram}+$ metribuzin & $0,47 \mathrm{~b}$ & $2,40 \mathrm{a}$ & $1,41 \mathrm{a}$ & $1,41 \mathrm{a}$ \\
\hline $2,4-\mathrm{D} /$ picloram + metribuzin & $0,00 \mathrm{~b}$ & $2,44 \mathrm{a}$ & $1,13 \mathrm{a}$ & $1,27 \mathrm{a}$ \\
\hline $2,4-\mathrm{D} /$ picloram + metribuzin & $0,47 \mathrm{~b}$ & $1,71 \mathrm{a}$ & 1,13 a & 1,41 a \\
\hline Imazapyr & $1,79 \mathrm{ab}$ & 2,94 a & $1,27 \mathrm{a}$ & $1,41 \mathrm{a}$ \\
\hline Metribuzin & $1,68 \mathrm{ab}$ & $2,23 \mathrm{a}$ & $1,27 \mathrm{a}$ & $1,41 \mathrm{a}$ \\
\hline Testemunha & $3,12 \mathrm{a}$ & $2,54 \mathrm{a}$ & $1,41 \mathrm{a}$ & $1,41 \mathrm{a}$ \\
\hline$F$ trat. & $4,26 * *$ & $1,01 \mathrm{NS}$ & $0,82 \mathrm{NS}$ & $1,00 \mathrm{NS}$ \\
\hline F bloco & $1,30 \mathrm{NS}$ & $1,59 \mathrm{NS}$ & $0,76 \mathrm{NS}$ & $1,00 \mathrm{NS}$ \\
\hline DMS & 2,42 & 1,74 & 0,62 & 0,20 \\
\hline CV $(\%)$ & 106,3 & 23,6 & 16,5 & 4,9 \\
\hline
\end{tabular}

'Médias seguidas de mesma letra não diferem significativamente entre si, pelo teste de Tukey ao nível de 5\% de probabilidade; NS: não significativo pelo teste $\mathrm{F}$; **: significativo ao nível de $1 \%$ de probabilidade pelo teste $\mathrm{F}$. 


\title{
CONTROLE QUÍMICO DE PARREIRA-BRAVA (Cissampelos glaberrima) ${ }^{1}$
}

\author{
Edson D. de Mattos ${ }^{2}$, Pedro Luís da Costa A. Alves ${ }^{3}$, Fabíola V. Môro ${ }^{3}$, José Custódio de Souza ${ }^{4}$, \\ José V. F. Martins ${ }^{5}$ e Francisco Rigler Neto ${ }^{5}$
}

\author{
'Parte do trabalho de graduação do primeiro autor à FCAVJ/UNESP \\ ${ }^{2}$ Graduando. FCAVJ/UNESP. Jaboticabal, SP 14870-000 \\ ${ }^{3}$ Professor, Dr. FCAV/UNESP plalves@fcav.unesp.br \\ ${ }^{4}$ Técnico Agrícola, Estagiário. FCAVJ/UNESP \\ ${ }^{5}$ Técnico de Laboratório. FCAVJ/UNESP
}

\begin{abstract}
RESUMO
O trabalho teve por objetivo encontrar uma alternativa para o controle químico da parreira-brava, uma planta daninha que vem se constituindo em sério problema para a cultura da cana-de-açúcar. Os herbicidas utilizados em pós-emergência foram glyphosate, isolado e em mistura em tanque com carfentrazone e com sulfentrazone, metribuzin, 2,4-D+diuron, acetochlor, ametryne e oxyfluorfen em mistura com diuron e com acetochlor. Em pré-emergência foram testadas as misturas oxyfluorfen+diuron, oxyfluorfen+2,4-D/picloram, oxyfluorfen+clomazone, oxyfluorfen+imazapyr, oxyfluorfen+carfentrazone oxyfluorfen+sulfentrazone, 2,4-D/picloram, clomazone+imazapyr, diuron/hexazinone+MSMA, e o tebuthiuron isolado, todos em suas doses recomendadas. Um terceiro ensaio foi realizado sob condições semicontroladas, visando o controle em pré-emergência, no qual os rizomas foram colocados em duas profundidades diferentes, 30 e $55 \mathrm{~cm}$, em tubos de PVC com capacidade de $42 \mathrm{l}$ de solo e submetido aos seguintes tratamentos: 2,4-D/picloram (1200/320, 1440/384 e 1680/448 g/ha), 2,4-D/picloram+imazapyr $(1200 / 320+500,1200 / 320+750$ e 1200/320+1000 g/ha), 2,4-D/picloram+metribuzin (1200/ $320+1920,1200 / 320+2400$ e 1200/320+2880 g/ha), imazapyr (750 g/ha) e metribuzin (2400 g/ha). Como resultado do controle químico em pós-emergência, apenas o tratamento contendo a mistura oxyfluorfen+acetochlor proporcionou um bom controle durante os primeiros 30 dias após a aplicação, quando começou a perder eficiência. Os tratamentos em pré-emergência com 2,4-D/picloram e tebuthiuron proporcionaram excelente controle da planta daninha durante 90 dias, e os tratamentos com imazapyr em mistura com oxyfluorfen e com clomazone resultaram em excelente controle durante os primeiros 60 dias, após os quais a eficiência diminuiu. O tratamento com 2,4-D/picloram não causou intoxicação à cultura. Nenhum dos tratamentos em pré-emergência matou os rizomas que se encontravam até $55 \mathrm{~cm}$ de profundidade, mas o $2,4-\mathrm{D} /$ picloram causou mortalidade das brotações, mostrando ser o herbicida mais promissor no controle dessa planta daninha.
\end{abstract}

Palavras-chave: Menispermaceae, cana-de-açúcar, herbicidas.

\section{ABSTRACT \\ Chemical control of Cissampelos glaberrima}

The aim of this study was to find a chemical alternative for the control of $C$. glaberrima, a weed that has became a serious problem in sugar cane plantations. The post emergent herbicides tested included: glyphosate, by itself and mixed with carfentrazone or sulfentrazone, metribuzin, 2,4-D + diuron, acetochlor, ametryne and oxyfluorfen mixed with diuron and acetochlor. In preemergence, oxyfluorfen mixed with diuron, 2,4-D/picloram, clomazone, imazapyr, carfentrazone and 
sulfentrazone, tebuthiuron, 2,4-D/picloram, clomazone mixed with imazapyr, and diuron/hexazinone mixed with MSMA were tested at recommended rates. A third trial was setup under semi controlled conditions aiming at a pre emergence control of this plant. A PVC cylindrical container containing 421 of soil was used. The rhizomes were placed at two differents depths: 30 and $55 \mathrm{~cm}$. In relation to postemergence herbicide treatments only oxyfluorfen mixed with acetochlor showed good control during the first 30 days after application, after which this treatment started to lose effect. The pre emergent treatments with 2,4-D/picloram and tebuthiuron showed the best control over 90 days and the treatments with imazapyr mixed with oxyfluorfen and clomazone resulted in an excellent control during 60 days in preemergence. The treatment with 2,4-D/picloram did not cause toxicity to the crop. None of the preemergence treatments killed the rhizomes at $55 \mathrm{~cm}$ depth, but 2,4-D/picloram caused death of the new shoots, this being the most promising herbicide to control this weed.

Key words: Menispermaceae, sugar cane, herbicides.

\section{INTRODUÇÃO}

A parreira-brava (Cissampelos glaberrima A. St.-Hil.) é uma planta pertencente à família Menispermaceae (Braga, 1989) e a atenção que lhe tem sido dada se deve basicamente às suas propriedades medicinais (Barbosa Filho et al., 1997 a,b; Rodrigues, 1989; Lorenzi, 1982). Contudo, recentemente, esta planta tem sido encontrada freqüentemente infestando canaviais localizados nos municípios de Jaboticabal e Pradópolis, SP.

A presença desta planta nos canaviais tem preocupado os canavicultores e os técnicos envolvidos com a cultura, por ser uma planta trepadeira lenhosa com um sistema radicular vigoroso (Barroso, 1978; Lorenzi, 1982; Braga, 1989). A parreira-brava tem dificultado os tratos culturais, prejudicando o crescimento da cultura (por enrolar nas folhas, fechando-as, e por dobrar o ápice do colmo) e, provavelmente, competindo com a cultura. Além disso, o controle químico até então utilizado pelas usinas e pelos fornecedores de cana-de-açúcar, integrado ou não a outras formas de controle, não tem se mostrado eficiente para esta planta. Como conseqüência e devido ao fato dela se multiplicar tanto pelos rizomas como por sementes (Braga, 1989), essa planta tem se disseminado rapidamente pela região.

Sabe-se que um dos pontos mais críticos no processo produtivo da cana-de-açúcar, assim como de outras culturas, é, sem dúvida, a interferência negativa imposta pelas plantas daninhas. Essas plantas, quando presentes, podem competir por recursos limitantes do meio (água, luz e nutrientes), podem liberar substâncias alelopáticas, podem hospedar pragas e doenças comuns a cultura ou, ainda, interferir no rendimento da colheita (Pitelli, 1985), justificando plenamente a preocupação em controlá-las.

Considerando que esta planta está no início de sua dispersão pela região e tendo em vista a sua agressividade e o seu difícil controle, este trabalho objetivou avaliar a eficácia de diferentes herbicidas para o seu controle, em pré e pósemergência, procurando assim, fornecer informações para o desenvolvimento de manejo eficiente desta planta daninha.

\section{MATERIAL E MÉTODOS}

O presente trabalho constituiu-se de estudos de algumas alternativas para o controle químico dessa planta daninha, com dois experimentos conduzidos em área de produção da Fazenda Santa Cecília, em Córrego Rico, SP, e um experimento (utilizando rizomas coletados previamente nessa fazenda) conduzido sob condições semicontroladas, em área anexa ao Laboratório de Biologia e Manejo de Plantas Daninhas, do Departamento de Biologia Aplicada à Agropecuária da FCAV/UNESP, Campus de Jaboticabal.

\section{Controle químico em pós-emergência}

Este experimento foi conduzido na Fazenda Santa Cecília, no período de 21/05/1999 (aplicação dos herbicidas em pós-emergência da planta daninha e da cultura) a 21/07/ 1999 (avaliação aos 60 dias após a aplicação). A cultivar de cana-de-açúcar que estava plantada era a RB 802233, cuja área seria submetida à reforma ao término deste experimento.

O delineamento experimental utilizado foi o de blocos casualizados, com dez tratamentos em quatro repetições. Cada parcela experimental constou de cinco linhas de plantio de cana, espaçadas de $1,4 \mathrm{~m}$ e com $10 \mathrm{~m}$ de comprimento cada. Como medida de segurança, foi deixada uma linha de plantio de cada lado da parcela e $1 \mathrm{~m}$ nas extremidades das parcelas como bordadura.

Os tratamentos experimentais constaram de uma única aplicação de vários herbicidas isolados ou em mistura, em pós-emergência da planta daninha e da cultura, com uma testemunha sem aplicação de herbicida (Tabela 1). Por ocasião da aplicação, as plantas de cana encontravam-se com duas folhas totalmente expandidas e, em média, com $30 \mathrm{~cm}$ de altura; as plantas de parreira-brava estavam em vários estágios de crescimento. Nessa ocasião, foi feito um levantamento visual da cobertura das parcelas proporcionada pela planta daninha, utilizando-se quadros amostrais de $1 \times 1 \mathrm{~m}$, subdivididos a cada $0,1 \mathrm{~m}$, jogados quatro vezes, aleatoria- 
Tabela 1. Tratamentos aplicados em pós-emergência da parreira-brava. Jaboticabal, SP. 1999.

\begin{tabular}{|c|c|}
\hline Tratamento & $\overline{D o s e}$ (g/ha) \\
\hline 1. Glyphosate ${ }^{1}+$ carfentrazone $^{2}$ & $1440+50$ \\
\hline 2. Glyphosate+sulfentrazone $e^{3}$ & $1440+500$ \\
\hline 3. Glyphosate ${ }^{4}$ & 1080 \\
\hline 4. Metribuzin ${ }^{5}$ & 960 \\
\hline 5. $2,4-\mathrm{D}^{6}+$ diuron $^{7}$ & $670+1600$ \\
\hline 6. Acetochlor ${ }^{8}$ & 1800 \\
\hline 7. Ametryne ${ }^{9}$ & 2000 \\
\hline 8. Oxyfluorfen ${ }^{10}+$ diuron & $240+1600$ \\
\hline 10. Oxyfluorfen+acetochlor & $480+1800$ \\
\hline 11. Testemunha & - \\
\hline
\end{tabular}

${ }^{1}$ Roundup WG, ${ }^{2}$ Aurora, ${ }^{3}$ Boral, ${ }^{4}$ Roundup CS, ${ }^{5}$ Sencor, ${ }^{6}$ DMA 806 BR, ${ }^{7}$ Karmex, ${ }^{8}$ Fist, ${ }^{9}$ Gesapax, ${ }^{10} \mathrm{Goal}$.

mente, na área útil das parcelas. O resultado, expresso em porcentagem da área, correspondeu a média das quatro jogadas.

A aplicação dos produtos foi dirigida à planta daninha e foi realizada com pulverizador costal, à pressão constante (ar comprimido), munido de um bico Teejet 8002 EVS e regulado para um gasto de volume de calda de $200 \mathrm{l} / \mathrm{ha}$. Essa aplicação iniciou-se às 10:00h e terminou às 11:00h. A temperatura do ar era de $22^{\circ} \mathrm{C}$ e a do solo $21^{\circ} \mathrm{C}$. O solo estava seco e a umidade relativa do ar era de $46 \%$, com ventos de até $2 \mathrm{~km} / \mathrm{h}$, sob um céu descoberto $(0 / 8)$.

Aos 15, 30, 45 e 60 dias após a aplicação, foram realizadas avaliações visuais de controle da parreira-brava, utilizando-se a escala de notas da ALAM (1974). Os dados obtidos foram transformados para arc sen $\sqrt{\%}$, submetidos a análise de variância pelo teste $\mathrm{F}$ e as médias foram comparadas pelo teste de Tukey ao nível de $5 \%$ de probabilidade.

\section{Controle químico em pré-emergência}

Este experimento também foi instalado e conduzido na Fazenda Santa Cecília, no período de 24/02/2000 (aplicação dos herbicidas na pré-emergência da planta daninha e da cultura) a 25/05/2000 (avaliação aos 90 dias após a aplicação). A área era de cana-soca, cultivar RB 825336, de quarto corte, colhida mecanicamente, mas com a palha sendo enleirada antes da aplicação dos herbicidas. O solo da área era um Latossolo Vermelho-Escuro, distrófico e A moderado. A análise granulométrica de uma amostra composta deste solo revelou que ele continha $300 \mathrm{~g} / \mathrm{kg}$ de argila, $60 \mathrm{~g} / \mathrm{kg}$ de limo, $340 \mathrm{~g} / \mathrm{kg}$ de areia fina e $300 \mathrm{~g} / \mathrm{kg}$ de areia grossa, sendo enquadrado na classe textural média.

O delineamento experimental utilizado foi o de blocos casualizados com doze tratamentos em quatro repetições. Cada parcela experimental constou de seis linhas de plantio de cana espaçadas de $1,4 \mathrm{~m}$ e com $40 \mathrm{~m}$ de comprimento cada. Como medida de segurança, foi deixada uma linha de plantio de cada lado da parcela e $5 \mathrm{~m}$ nas extremidades das parcelas como bordadura.

Os tratamentos experimentais constaram de uma única aplicação de vários herbicidas, isolados ou em mistura, em pré-emergência da planta daninha e da cultura, com uma testemunha sem aplicação de herbicida (Tabela 2).

A aplicação dos produtos foi realizada com pulverizador costal à pressão constante (ar comprimido), munido de barra com 4 bicos XR 11002 e regulado para um consumo de calda de $200 \mathrm{l} / \mathrm{h}$. A aplicação iniciou-se às 9:50 h e terminou às 12:00 h. A temperatura do ar foi de $31,5^{\circ} \mathrm{C}$ e a do solo, a dez centímetros, foi de $25^{\circ} \mathrm{C}$. O solo estava seco e a umidade relativa do ar era de $53 \%$, com ventos de até $2 \mathrm{~km} / \mathrm{h}$, sob um céu parcialmente coberto (2/8).

Para detectar efeitos tóxicos dos produtos na cultura, aos 90 dias após a aplicação foram realizadas avaliações de altura (medida até a última lígula visível), contagem do número de folhas, área foliar (Li-Cor, mod. LI 3000A), biomassa seca dos colmos e folhas (obtidas após $96 \mathrm{~h}$ de secagem em estufa com circulação forçada de ar a $70^{\circ} \mathrm{C}$ ).

Na população de parreira-brava foram realizadas avaliações visuais de controle, utilizando-se a escala de notas da Asociacion Latinoamericana de Malezas (1974). Essas avaliações foram realizadas aos 30,60 e 90 dias após a aplicação.

Tabela 2. Tratamentos aplicados em pré-emergência da parreira-brava. Jaboticabal, SP. 2000.

\begin{tabular}{|c|c|}
\hline Tratamento & Dose (g/ha) \\
\hline 1. Oxyfluorfen ${ }^{1}+$ diuron $^{2}$ & $720+2400$ \\
\hline 2. Oxyfluorfen + diuron & $1080+2800$ \\
\hline 3. Oxyfluorfen $+2,4 \mathrm{D} /$ picloram $^{3}$ & $720+720 / 192$ \\
\hline 4. Oxyfluorfen + clomazone $^{4}$ & $720+1250$ \\
\hline 5. Oxyfluorfen + imazapyr $^{5}$ & $720+625$ \\
\hline 6. Oxyfluorfen + carfentrazone $^{6}$ & $720+150$ \\
\hline 7. Tebuthiuron ${ }^{7}$ & 1250 \\
\hline 8. 2,4-D/ picloram & $1200 / 320$ \\
\hline 9. Clomazone + imazapyr & $1250+625$ \\
\hline 10. Oxyfluorfen + sulfentrazone $^{8}$ & $720+750$ \\
\hline 11. Diuron/hexazinone ${ }^{9}+\mathrm{MSMA}^{10}$ & $360 / 102+1920$ \\
\hline 12. Testemunha & - \\
\hline
\end{tabular}

${ }^{1}$ Goal, ${ }^{2}$ Karmex, ${ }^{3}$ Tordon, ${ }^{4}$ Gamit, ${ }^{5}$ Contain, ${ }^{6}$ Aurora, ${ }^{7}$ Combine, ${ }^{8}$ Boral, "VelparK, ${ }^{11}$ Daconate. 
Os dados obtidos foram submetidos a análise de variância pelo teste $\mathrm{F}$ e as médias foram comparadas pelo teste de Tukey ao nível de $5 \%$ de probabilidade, sendo que os dados de controle foram previamente transformados para arc sen $\sqrt{\%}$.

\section{Controle químico em pré-emergência, sob condições semicontroladas.}

Este experimento foi instalado e conduzido no período de 09/03/2000 (aplicação dos herbicidas na pré-emergência da planta daninha) a 08/05/2000 (avaliação aos 60 dias após a aplicação), em área anexa ao Laboratório de Biologia e Manejo de Plantas Daninhas, sob condições semicontroladas, ou seja, sem limitação de água.

O substrato foi coletado na camada arável $(0-20 \mathrm{~cm})$ de um solo Latossolo Vermelho-Escuro, distrófico e A moderado. A análise granulométrica de uma amostra composta deste solo revelou que ele continha $290 \mathrm{~g} / \mathrm{kg}$ de argila, $60 \mathrm{~g} /$ $\mathrm{kg}$ de limo, $340 \mathrm{~g} / \mathrm{kg}$ de areia fina e $310 \mathrm{~g} / \mathrm{kg}$ de areia grossa, sendo enquadrado na classe textural média. Esse solo foi seco à sombra, peneirado e acondicionado em tubos de PVC com $30 \mathrm{~cm}$ de diâmetro por $60 \mathrm{~cm}$ de altura, perfazendo $421 \mathrm{de}$ capacidade, com a base tampada com tela tipo mosquiteiro.

Por ocasião do preenchimento dos tubos, foram colocados dois rizomas próximos à base, a $55 \mathrm{~cm}$ de profundidade, e mais dois a $30 \mathrm{~cm}$ de profundidade. Esse procedimento foi adotado com o intuito de verificar se os herbicidas, de acordo com as suas capacidades de percolação, atingiriam esses rizomas. Após o plantio dos rizomas até o término do período experimental, procurou-se manter a umidade do solo mediante irrigações periódicas com água, de forma que os solos de todos os tubos ficassem visualmente úmidos.

$\mathrm{O}$ delineamento experimental utilizado foi o de blocos casualizados com doze tratamentos e três repetições. Cada parcela experimental constou de um tubo de PVC.

Sete dias após o plantio dos rizomas, foram aplicados os tratamentos. Estes constaram de uma única aplicação, em pré-emergência, dos herbicidas que apresentaram os melhores resultados no experimento em campo, particularmente o 2,4-D/picloram, isolado ou em mistura com herbicidas de elevada solubilidade em água, com uma testemunha sem aplicação de herbicida (Tabela 3).

A aplicação dos produtos foi realizada com pulverizador um costal, à pressão constante (ar comprimido), munido de barra com dois bicos XR 11002 e regulado para um consumo de calda de $200 \mathrm{l} / \mathrm{ha}$. A aplicação iniciou-se às 9:00 h e terminou às $9: 20 \mathrm{~h}$. A temperatura do ar era de $26,5^{\circ} \mathrm{C}$ e a do solo $22,3^{\circ} \mathrm{C}$. O solo estava úmido e a umidade relativa do ar era de $68 \%$, com ventos de até $4 \mathrm{~km} / \mathrm{h}$, porém em rajadas, sob um céu pouco coberto (1/8).

Aos 60 dias após a aplicação, embora praticamente nenhuma brotação tivesse emergido, o experimento foi desmontado e os rizomas retirados dos tubos com o auxílio de jatos d'água. A avaliação dos efeitos dos herbicidas foi feita
Tabela 3. Tratamentos aplicados em pré-emergência da parreira-brava, sob condições semicontroladas. Jaboticabal, SP. 2000.

\begin{tabular}{|c|c|}
\hline Tratamento & Dose (g/ha) \\
\hline 1. Oxyfluorfen ${ }^{1}+$ diuron $^{2}$ & $720+2400$ \\
\hline 2. Oxyfluorfen+diuron & $1080+2800$ \\
\hline 3. Oxyfluorfen $+2,4 \mathrm{D} /$ picloram $^{3}$ & $720+720 / 192$ \\
\hline 4. Oxyfluorfen+clomazone ${ }^{4}$ & $720+1250$ \\
\hline 5. Oxyfluorfen + imazapyr ${ }^{5}$ & $720+625$ \\
\hline 6. Oxyfluorfen+carfentrazone ${ }^{6}$ & $720+150$ \\
\hline 7. Tebuthiuron? & 1250 \\
\hline 8. 2,4-D/ picloram & $1200 / 320$ \\
\hline 9. Clomazone+imazapyr & $1250+625$ \\
\hline 10. Oxyfluorfen+sulfentrazone ${ }^{8}$ & $720+750$ \\
\hline 11. Diuron/hexazinone ${ }^{9}+\mathrm{MSMA}^{10}$ & $360 / 102+1920$ \\
\hline 12. Testemunha & - \\
\hline
\end{tabular}

${ }^{1}$ Tordon, ${ }^{2}$ Contain, ${ }^{3}$ Sencor.

contando-se o número de brotações vivas emitidas por rizoma e o número de rizomas vivos. A mortalidade do rizoma foi avaliada em função da turgidez e por observações visuais de coloração, após ser submetido a vários cortes longitudinais e transversais, sendo considerado morto aquele que se apresentava pouco túrgido (murcho) e com coloração interna escura.

Os dados de contagem obtidos foram transformados para $\sqrt{n}$, submetidos a análise de variância pelo teste $F$ e as médias foram comparadas pelo teste de Tukey ao nível de $5 \%$ de probabilidade.

\section{RESULTADOS E DISCUSSÃO}

\section{Controle químico em pós-emergência}

A cobertura visual das parcelas experimentais pela parreira-brava foi, em média, de $22 \%$. Aos 15 dias após a aplicação (DAA) dos tratamentos, considerando a avaliação inicial de cobertura, observou-se que o glyphosate em mistura com carfentrazone ou com sulfentrazone ou mesmo isolado e o acetochlor sozinho (tratamentos 1, 2, 3 e 6, respectivamente) proporcionaram um controle ruim (nota 1), resultado este que se manteve até o final do período experimental, aos 60 DAA (Tabela 4). O tratamento com aplicação de metribuzin (tratamento 4) proporcionou um controle inicialmente ruim (nota 1), resultado que evolui um pouco, passando para regular (nota 2) aos 30 DAA e assim se manteve até 
Controle químico de Parreira-Brava..

Tabela 4. Percentagens de controle químico em pós-emergência da parreira-brava aos 15, 30, 45 e 60 dias após a aplicação (DAA), com as respectivas notas visuais, nas diferentes épocas de avaliação. Jaboticabal, SP. 1999.

\begin{tabular}{|c|c|c|c|c|c|c|c|c|c|c|c|c|}
\hline \multirow{2}{*}{$\begin{array}{l}\text { Trata- } \\
\text { mento }\end{array}$} & \multicolumn{3}{|c|}{15 DAA } & \multicolumn{3}{|c|}{30 DAA } & \multicolumn{3}{|c|}{$45 \mathrm{DAA}$} & \multicolumn{3}{|c|}{60 DAA } \\
\hline & D.O. & D.T. & $\overline{\text { Nota }}$ & D.O. & D.T. & $\overline{\text { Nota }}$ & D.O. & D.T. & Nota & D.O. & D.T. & Nota \\
\hline 1 & 15 & $22,1 \mathrm{ef}^{1}$ & 1 & 12 & 17,9 ef & 1 & 15 & $19,9 \mathrm{~cd}$ & 1 & 16 & $17,9 \mathrm{~cd}$ & 1 \\
\hline 2 & 20 & 26,2 de & 1 & 15 & 19,0 ef & 1 & 5 & $6,6 \mathrm{~cd}$ & 1 & 7 & $11,2 \mathrm{~cd}$ & 1 \\
\hline 3 & 5 & $6,6 \mathrm{fg}$ & 1 & 7 & $11,2 \mathrm{ef}$ & 1 & 10 & $9,8 \mathrm{~cd}$ & 1 & 7 & $11,2 \mathrm{~cd}$ & 1 \\
\hline 4 & 37 & 37,7 cde & 1 & 47 & $43,5 \mathrm{~cd}$ & 2 & 50 & $45,1 \mathrm{ab}$ & 2 & 47 & $43,5 \mathrm{ab}$ & 2 \\
\hline 5 & 47 & 43,5 bcd & 2 & 57 & 49,4 bcd & 2 & 60 & $50,9 \mathrm{a}$ & 2 & 55 & $47,9 \mathrm{a}$ & 2 \\
\hline 6 & 37 & 37,4 cde & 1 & 25 & 29,9 de & 1 & 17 & $24,5 \mathrm{bc}$ & 1 & 17 & $24,5 \mathrm{bc}$ & 1 \\
\hline 7 & 60 & $51,0 \mathrm{abc}$ & 2 & 67 & $55,5 \mathrm{abc}$ & 3 & 55 & $47,9 \mathrm{a}$ & 2 & 50 & $45,0 \mathrm{a}$ & 2 \\
\hline 8 & 70 & $57,1 \mathrm{ab}$ & 3 & 87 & $69,2 \mathrm{ab}$ & 5 & 70 & 56,9 a & 3 & 65 & $53,8 \mathrm{a}$ & 3 \\
\hline 9 & 85 & $67,5 \mathrm{a}$ & 5 & 94 & $75,7 \mathrm{a}$ & 6 & 77 & $61,8 \mathrm{a}$ & 4 & 75 & $60,1 \mathrm{a}$ & 4 \\
\hline 10 & 0 & $0,0 \mathrm{~g}$ & 1 & 0 & $0,0 \mathrm{f}$ & 1 & 0 & $0,0 \mathrm{~d}$ & 1 & 0 & $0,0 \mathrm{~d}$ & 1 \\
\hline$F$ trat & & $32,20 * *$ & & & $32,78^{* *}$ & & & $22,44^{* *}$ & & & $27,18^{* *}$ & \\
\hline F Blocos & & $1,22 \mathrm{NS}$ & & & $0,97 \mathrm{NS}$ & & & $1,46 \mathrm{NS}$ & & & $0,98 \mathrm{NS}$ & \\
\hline DMS & & 18,4 & & & 21,6 & & & 23,3 & & & 19,6 & \\
\hline CV \% & & 21,7 & & & 24,0 & & & 29,7 & & & 25,5 & \\
\hline
\end{tabular}

D.O.:dados originais ; D.T.: dados transformados em arc sen $x$; DAA.

${ }^{1}$ Médias seguidas de mesma letra não diferem significativamente entre si, pelo teste de Tukey ao nível de 5\% de probabilidade; NS: não significativo pelo teste $\mathrm{F}$; ${ }^{* *}$ : significativo ao nível de $1 \%$ de probabilidade pelo teste $\mathrm{F}$.

os 60 DAA. A mistura 2,4-D+diuron e o ametryne (tratamentos 5 e 7 , respectivamente) proporcionaram um controle regular (nota 2) dos 15 aos 60 DAA. Os tratamentos que utilizaram oxyfluorfen em mistura com diuron ou acetochlor (tratamentos 9 e 10) proporcionaram os melhores resultados, com controle suficiente (nota 3 ) e bom (nota 4) da planta daninha aos $15 \mathrm{DAA}$, que evoluiu um pouco aos $30 \mathrm{DAA}$, quando proporcionaram um controle muito bom (nota 5) e excelente (nota 6), respectivamente. Mas, aos 45 DAA, ocorreu redução da eficiência, quando o controle passou para suficiente (nota 3) e bom (nota 4), e assim se manteve até os 60 DAA.

\section{Controle químico em pré-emergência}

Os resultados das determinações de altura, número de folhas, área foliar, biomassa seca dos colmos e das folhas, realizadas nas plantas de cana-de-açúcar aos 90 dias após a aplicação (DAA), encontram-se na Tabela 5. Analisando-se os dados, constatou-se que o tratamento com aplicação de 2,4-D/picloram (tratamento 8) e os tratamentos com imazapyr (tratamentos 5 e 9) proporcionaram os maiores e menores resultados, respectivamente, quanto aos parâmetros avaliados, em comparação com os demais tratamentos e à testemunha. O tratamento com 2,4-D/picloram e os tratamentos com imazapyr, resultaram respectivamente, em plantas com aproximadamente 31,5 e 20,0 cm de altura, 5,5 e 4,7 folhas, 663,03 e $311,17 \mathrm{~cm}^{2}$ de área foliar, 128,36 e 41,34 $\mathrm{g}$ de matéria seca de colmos e 92,54 e $38,06 \mathrm{~g}$ de matéria seca de folhas, en- quanto os demais tratamentos resultaram em plantas com aproximadamente em $25,9 \mathrm{~cm}$ de altura, 5,0 folhas, 519,16 $\mathrm{cm}^{2}$ de área foliar, 79,21 $\mathrm{g}$ de matéria seca de colmos e 62,25 $\mathrm{g}$ de matéria seca de folhas.

Com relação ao controle em pré-emergência da planta daninha (Tabela 6), verificou-se, aos 30 dias após a aplicação, que as misturas de oxyfluorfen com diuron, nas doses de $720+2400$ e $1080+2800 \mathrm{~g} / \mathrm{ha}$, proporcionaram um controle regular (nota 2) e muito bom (nota 5), respectivamente. Os tratamentos que envolveram as misturas de oxyfluorfen com 2,4-D/picloram, oxyfluorfen com imazapyr, 2,4-D/picloram, clomazone com imazapyr e oxyfluorfen com sulfentrazone proporcionaram excelente controle (nota 6). A mistura de oxyfluorfen com clomazone na dose de $720+1250 \mathrm{~g} /$ ha proporcionou um controle regular (nota 2), enquanto a mistura do oxyfluorfen com carfentrazone resultou num controle muito bom (nota 5). O tebuthiuron na dose de $1250 \mathrm{~g} /$ ha resultou em bom controle (nota 4), aos 15 DAA. Todos os produtos testados apresentaram resultado superior à mistura de diuron/ hexazinone com MSMA, tratamento utilizado pela Fazenda Santa Cecília no controle dessa planta daninha, que resultou em nenhum controle (nota 1) aos 30 DAA.

Aos 60 DAA, o oxyfluorfen em mistura com diuron, na menor dose, manteve a eficácia observada aos 30 DAA, com um controle regular (nota 2). Já o oxyfluorfen com diuron, na maior dose, e com 2,4-D/picloram, apresentaram perda de eficácia quando o controle passou de muito bom (nota 5) e excelente (nota 6), respectivamente, para regular (nota 2). As misturas oxyfluorfen com clomazone e com carfentrazone, 
Pedro Luís da C. A. Alves et al.

Tabela 5. Efeitos dos herbicidas, aplicados em pré-emergência, sobre a altura, número de folhas, área foliar e biomassa seca de colmos e folhas das plantas de cana-de-açúcar, avaliadas aos 90 dias após a aplicação. Jaboticabal, 2000.

\begin{tabular}{|c|c|c|c|c|c|}
\hline \multirow{2}{*}{ Tratamento } & \multirow{2}{*}{$\begin{array}{c}\text { Altura } \\
(\mathrm{cm})\end{array}$} & \multirow{2}{*}{$\begin{array}{c}\text { Número } \\
\text { de folhas }{ }^{2}\end{array}$} & \multirow{2}{*}{$\begin{array}{c}\text { Área foliar }{ }^{2} \\
\left(\mathrm{~cm}^{2}\right)\end{array}$} & \multicolumn{2}{|c|}{ Biom assa $\sec ^{2}(\mathrm{~g})$} \\
\hline & & & & Colmo & Folha \\
\hline 1 & 4,6 ef $^{1}$ & $2,3 \mathrm{ab}$ & $18,33 \mathrm{de}$ & $6,93 \mathrm{fg}$ & $6,51 \mathrm{de}$ \\
\hline 2 & $4,7 \mathrm{e}$ & $2,2 \mathrm{ab}$ & $19,60 \mathrm{c}$ & $6,96 \mathrm{fg}$ & 6,47 ef \\
\hline 3 & $5,0 \mathrm{~cd}$ & $2,3 \mathrm{ab}$ & $22,86 \mathrm{~b}$ & $8,63 \mathrm{cde}$ & $8,10 \mathrm{c}$ \\
\hline 4 & $5,0 \mathrm{~cd}$ & $2,3 \mathrm{ab}$ & $22,80 \mathrm{~b}$ & $8,49 \mathrm{de}$ & $7,89 \mathrm{c}$ \\
\hline 5 & 4,6 ef & $2,3 \mathrm{ab}$ & $17,64 \mathrm{e}$ & $6,93 \mathrm{fg}$ & 6,41 ef \\
\hline 6 & $4,9 d$ & $2,1 \mathrm{~b}$ & $18,71 \mathrm{cde}$ & $8,13 \mathrm{e}^{-}$ & $6,83 \mathrm{~d}$ \\
\hline 7 & $4,9 \mathrm{~d}$ & $2,2 \mathrm{ab}$ & $19,17 \mathrm{~cd}$ & $7,42 \mathrm{f}$ & $6,80 \mathrm{~d}$ \\
\hline 8 & 5,6 a & $2,3 \mathrm{ab}$ & 25,75 a & $11,33 \mathrm{a}$ & 9,62 a \\
\hline 9 & $4,5 \mathrm{f}$ & $2,2 \mathrm{~b}$ & $18,19 \mathrm{de}$ & $6,43 \mathrm{~g}$ & $6,17 \mathrm{f}$ \\
\hline 10 & $5,1 \mathrm{c}$ & $2,2 \mathrm{~b}$ & $22,92 \mathrm{~b}$ & $8,90 \mathrm{~cd}$ & $8,46 \mathrm{~b}$ \\
\hline 11 & $5,4 \mathrm{~b}$ & 2,4 a & $25,87 \mathrm{a}$ & $9,82 \mathrm{~b}$ & $8,75 \mathrm{~b}$ \\
\hline 12 & $5,1 \mathrm{~cd}$ & $2,3 \mathrm{ab}$ & $23,71 \mathrm{~b}$ & $9,17 \mathrm{c}$ & $8,65 \mathrm{~b}$ \\
\hline $\mathrm{F}$ tratamentos & $86,25 * *$ & $3,62 * *$ & $152,91 * *$ & $151,70 * *$ & $302,36 * *$ \\
\hline DMS & 0,2 & 0,2 & 1,20 & 0,57 & 0,33 \\
\hline CV $(\%)$ & 1,4 & 4,4 & 2,3 & 2,8 & 1,8 \\
\hline
\end{tabular}

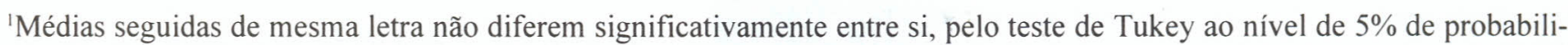
dade;

${ }^{2}$ Dados transformados para $\sqrt{\mathrm{n}}$

NS: não significativo pelo teste $\mathrm{F}$; **: significativo ao nível de $1 \%$ de probabilidade pelo teste $\mathrm{F}$.

Tabela 6. Percentagens de controle de parreira-brava aos 30,60 e 90 dia após a aplicação (DAA) em pré-emergência, com as respectivas notas visuais, nas diferentes épocas de avaliação. Jaboticabal, SP, 2000.

\begin{tabular}{|c|c|c|c|c|c|c|c|c|c|}
\hline \multirow{2}{*}{$\begin{array}{l}\text { Trata- } \\
\text { mento }\end{array}$} & \multicolumn{3}{|c|}{30 DAA } & \multicolumn{3}{|c|}{60 DAA } & \multicolumn{3}{|c|}{90 DAA } \\
\hline & D.O. & D.T. & Nota & D.O. & D.T. & Nota & D.O. & D.T. & Nota \\
\hline 1 & 60 & $50,8 \mathrm{e}^{1}$ & 2 & 50 & $45,0 \mathrm{~d}$ & 2 & 10 & 18,4 ef & 1 \\
\hline 2 & 90 & $71,8 \mathrm{c}$ & 5 & 60 & $50,8 \mathrm{~cd}$ & 2 & 20 & $26,1 \mathrm{e}$ & 1 \\
\hline 3 & 95 & $84,5 \mathrm{ab}$ & 6 & 60 & $50,8 \mathrm{~cd}$ & 2 & 50 & $45,0 \mathrm{~d}$ & 2 \\
\hline 4 & 50 & $45,0 \mathrm{e}$ & 2 & 20 & $26,2 \mathrm{e}$ & 1 & 5 & $10,4 \mathrm{fg}$ & 1 \\
\hline 5 & 100 & 90,0 a & 6 & 100 & 90,0 a & 6 & 71 & $57,4 \mathrm{c}$ & 4 \\
\hline 6 & 90 & $71,8 \mathrm{c}$ & 5 & 70 & $56,8 \mathrm{bc}$ & 3 & 40 & $39,2 \mathrm{~d}$ & 1 \\
\hline 7 & 80 & $63,4 \mathrm{~d}$ & 4 & 95 & 78,9 a & 6 & 100 & $90,0 \mathrm{a}$ & 6 \\
\hline 8 & 100 & 90,0 a & 6 & 100 & 90,0 a & 6 & 100 & $90,0 \mathrm{a}$ & 6 \\
\hline 9 & 100 & 90,0 a & 6 & 98 & 81,9 a & 6 & 91 & $72,6 \mathrm{~b}$ & 6 \\
\hline 10 & 95 & $78,9 \mathrm{bc}$ & 6 & 80 & $63,5 \mathrm{~b}$ & 4 & 40 & $39,2 \mathrm{~d}$ & 1 \\
\hline 11 & 0 & $0,0 \mathrm{f}$ & 1 & 10 & $15,8 \mathrm{a}$ & 1 & 0 & $0,0 \mathrm{~g}$ & 1 \\
\hline 12 & 0 & $0,0 \mathrm{f}$ & 1 & 0 & $0,0 \mathrm{f}$ & 1 & 0 & $0,0 \mathrm{~g}$ & 1 \\
\hline$F$ trat & & $363,65 * *$ & & & $152,80 * *$ & & & $219,85^{* *}$ & \\
\hline DMS & & 8,3 & & & 11,6 & & & 10,9 & \\
\hline CV $(\%)$ & & 5,5 & & & 8,7 & & & 9,1 & \\
\hline
\end{tabular}

D.O. :Dados originais; D.T.: dados transformados em arc sen $\sqrt{\mathrm{x}}$;

'Médias seguidas de mesma letra não diferem significativamente entre si, pelo teste de Tukey ao nível de 5\% de probabili dade;

NS: não significativo pelo teste $\mathrm{F}$; ${ }^{* *}$ : significativo ao nível de $1 \%$ de probabilidade pelo teste $\mathrm{F}$. 
também apresentaram perda de eficácia aos 60 DAA, passando de um controle regular (nota 2) e muito bom (nota 5), respectivamente, para nenhum controle (nota 1) e controle suficiente (nota 3 ). Os tratamentos de imazapyr em mistura com oxyfluorfen e com clomazone, e o tratamento com 2,4D/picloram, continuaram proporcionando um excelente controle (nota 6) aos 60 DAA. O tratamento com tebuthiuron apresentou aumento de eficácia aos $60 \mathrm{DAA}$, passando de controle bom (nota 4) para excelente controle (nota 6). Já o tratamento que continha a mistura de oxyfluorfen com sulfentrazone mostrou perda de eficácia, com o controle passando de excelente (nota 6) para bom (nota 4). O tratamento padrão, diuron/hexazinone com MSMA, continuou com nenhum controle (nota 1).

Aos 90 DAA, os tratamentos continuaram apresentando perda de eficácia. Os tratamentos com oxyfluorfen em mistura com os produtos diuron, na menor dose, 2,4-D/ picloram, clomazone, carfentrazone e sulfentrazone, e o tratamento que continha a mistura diuron/hexazinone com MSMA, não proporcionaram qualquer controle (nota 1). Já o oxyfluorfen em mistura com diuron, na maior dose, e em mistura com imazapyr, proporcionou um controle regular (nota 2) e um controle bom (nota 4), respectivamente. Os tratamentos que continham 2,4-D/picloram, ou tebuthiuron, ou clomazone com imazapyr proporcionaram excelente controle (nota 6), conforme observado na Tabela 6 .

\section{Controle químico em pré-emergência, sob condições} semicontrolas.
Neste experimento, foram utilizados os herbicidas aplicados em pré-emergência que haviam apresentado os melhores resultados em condição de campo, particularmente 2,4-D/picloram, isolado ou em mistura com produtos com elevada solubilidade em água, para se observar a eficácia desses produtos no controle de rizomas de Cissampelos glaberrima nas profundidades de 30 e $55 \mathrm{~cm}$.

Analisando os resultados contidos na Tabela 7, observa-se que quanto ao número de brotações vivas nos rizomas que se encontravam a $30 \mathrm{~cm}$, todos os tratamentos que continham 2,4-D/picloram, tanto isolado quanto em mistura com imazapyr ou com metribuzin, independente da dose utilizada, proporcionaram os melhores resultados, ou seja, estavam com menor número de brotações viáveis. Quando se utilizou imazapyr ou metribuzin isolados, os resultados obtidos não foram satisfatórios.

Quanto ao número de rizomas viáveis encontrados a $30 \mathrm{~cm}$, pode-se observar que não houve diferenças significativas, evidenciando que os produtos, mesmo aqueles que controlaram as brotações, não mataram os rizomas, o que apenas retardaria o surgimento desta planta daninha.

Quando os rizomas de parreira-brava encontravam-se na profundidade de $55 \mathrm{~cm}$, verificou-se que nenhum dos produtos testados, em qualquer dose e qualquer mistura, reduziu significativamente tanto o número de brotações viáveis, como o de rizomas viáveis, o que evidencia que esses produtos não apresentaram mobilidade no solo suficiente para atingir os rizomas que se encontravam nesta profundidade ou, se os atingiram, não foi em concentração suficiente para matá-los.

Tabela 7. Número de brotações viáveis por rizoma e de rizomas viáveis de parreira-brava, nas profundidades de 30 e $55 \mathrm{~cm}$, em resposta aos tratamentos. Jaboticabal, 2000.

\begin{tabular}{|c|c|c|c|c|c|c|c|c|c|}
\hline \multirow{2}{*}{$\begin{array}{l}\text { Trata- } \\
\text { mento }\end{array}$} & \multicolumn{3}{|c|}{30 DAA } & \multicolumn{3}{|c|}{60 DAA } & \multicolumn{3}{|c|}{90 DAA } \\
\hline & D.O. & D.T. & Nota & D.O. & D.T. & nota & D.0. & D.T. & Nota \\
\hline 1 & 60 & $50,8 \mathrm{e}^{1}$ & 2 & 50 & $45,0 \mathrm{~d}$ & 2 & 10 & 18,4 ef & 1 \\
\hline 2 & 90 & $71,8 \mathrm{c}$ & 5 & 60 & $50,8 \mathrm{~cd}$ & 2 & 20 & $26,1 \mathrm{e}$ & 1 \\
\hline 3 & 95 & $84,5 \mathrm{ab}$ & 6 & 60 & $50,8 \mathrm{~cd}$ & 2 & 50 & $45,0 \mathrm{~d}$ & 2 \\
\hline 4 & 50 & $45,0 \mathrm{e}$ & 2 & 20 & $26,2 \mathrm{e}$ & 1 & 5 & $10,4 \mathrm{fg}$ & 1 \\
\hline 5 & 100 & $90,0 \mathrm{a}$ & 6 & 100 & 90,0 a & 6 & 71 & $57,4 \mathrm{c}$ & 4 \\
\hline 6 & 90 & $71,8 \mathrm{c}$ & 5 & 70 & $56,8 \mathrm{bc}$ & 3 & 40 & $39,2 \mathrm{~d}$ & 1 \\
\hline 7 & 80 & $63,4 \mathrm{~d}$ & 4 & 95 & 78,9 a & 6 & 100 & $90,0 \mathrm{a}$ & 6 \\
\hline 8 & 100 & 90,0 a & 6 & 100 & $90,0 \mathrm{a}$ & 6 & 100 & 90,0 a & 6 \\
\hline 9 & 100 & $90,0 \mathrm{a}$ & 6 & 98 & 81,9 a & 6 & 91 & $72,6 \mathrm{~b}$ & 6 \\
\hline 10 & 95 & $78,9 \mathrm{bc}$ & 6 & 80 & $63,5 \mathrm{~b}$ & 4 & 40 & $39,2 \mathrm{~d}$ & 1 \\
\hline 11 & 0 & $0,0 \mathrm{f}$ & 1 & 10 & $15,8 \mathrm{a}$ & 1 & 0 & $0,0 \mathrm{~g}$ & 1 \\
\hline 12 & 0 & $0,0 \mathrm{f}$ & 1 & 0 & $0,0 \mathrm{f}$ & 1 & 0 & $0,0 \mathrm{~g}$ & 1 \\
\hline F trat & & $363,65 * *$ & & & $152,80 * *$ & & & $219,85^{* *}$ & \\
\hline DMS & & 8,3 & & & 11,6 & & & 10,9 & \\
\hline CV $(\%)$ & & 5,5 & & & 8,7 & & & 9,1 & \\
\hline
\end{tabular}

${ }^{1}$ Médias seguidas de mesma letra não diferem significativamente entre si, pelo teste de Tukey ao nível de 5\% de probabilidade;

NS: não significativo pelo teste $\mathrm{F} ;{ }^{* *}$ : significativo ao nível de $1 \%$ de probabilidade pelo teste $\mathrm{F}$. 
Como no campo foram encontrados vários rizomas nesta profundidade, isso significa que poderá haver uma ressurgência da planta daninha e o controle químico por si só pode não ser suficiente para controlá-la, necessitando, portanto, da integração com outra forma de controle, como, por exemplo, o mecânico.

Dentre os herbicidas aplicados em pré-emergência, o fato do 2,4-D/picloram ter resultado no melhor controle da parreira-brava pode ser devido à sua elevada persistência no solo. Segundo Rodrigues \& Almeida (1998), o componente da mistura picloram é de alta persistência no solo, necessitando de um intervalo de dois a três anos antes de o terreno ser usado por culturas dicotiledôneas. Ainda, segundo esses autores, a solubilidade do picloram em água é infinita e ele é fracamente adsorvido à matéria orgânica e à argila, o que facilita sua percolação no solo. Por outro lado, o componente 2,4-D éster da formulação é praticamente insolúvel, ficando fortemente adsorvido aos colóides do solo.

\section{CONCLUSÕES}

O controle químico da parreira-brava (Cissampelos glaberrima) em pós-emergência mostrou-se ineficaz, enquanto o controle em pré-emergência, com 2,4-D/picloram (1200/ $320 \mathrm{~g} / \mathrm{ha}$ ) foi excelente até os 90 dias após a aplicação, sem causar intoxicação à cultura da cana-de açúcar. Nenhum dos herbicidas aplicados em pré-emergência matou os rizomas dessa planta daninha.

\section{LITERATURA CITADA}

ASOCIACION LATINOAMERICANA DE MALEZAS. Recomendaciones de los sistemas de evaluación en ensayos de control de malezas. ALAM, Bogotá, v.1, n.1, p.35-38. 1974.

BARBOSA FILHO, J.M.; AGRA, M.A.; THOMAS, G. Botanical, chemical and pharmacological investigation on Cissampelos species from Paraíba (Brazil). Ciência e Cultura, v. 49, n. 5/6, p.386-394. 1997 a.

BARBOSA FILHO, J.M.; CUNHA, E.V.L.; CORNÉLIO, M.L.; DIAS, C.S.; AL GRAY. Cissaglaberrimine, an aporphine alkaloid from Cissampelos glaberrima. Phytochemistry, v. 44, n. 4, p.959-961. 1997 b.

BARRoSO, G.M. Sistemática de Angiospermas do Brasil. V.1. Piracicaba: USP/ESALQ, 1978, 255p.

BRAGA, R. Plantas do Nordeste, especialmente do Ceará. Natal: Editora Universitária/UFRN, 1988, 540p.

LORENZI, H. Plantas daninhas do Brasil: terrestres, aquáticas, parasitas, tóxicas e medicinais. Nova Odessa: $\mathrm{H}$. Lorenzi (ed.), 1982, 425p.

PITELLI, R.A. Interferência de plantas daninhas em culturas agrícolas. Inf. Agropec., v. 11, n.129, p.16-27. 1985.

RODRIGUES, R.M. A flora Amazônica. Belém: CEJUP, $1989,462 \mathrm{p}$. 